\title{
Measuring childhood vaccine coverage in England: the role of Child Health Information Systems
}

G Amirthalingam (gayatri.amirthalingam@hpa.org.uk) ${ }^{1}$, J White', M Ramsay ${ }^{1}$

1. Immunisation, Hepatitis and Blood Safety Department, Health Protection Services, Health Protection Agency, London, United Kingdom

Amirthalingam G, White J, Ramsay M. Measuring childhood vaccine coverage in England: the role of Child Health Information Systems . Euro Surveill. 2012;17(16):pii=20149. Available online: http://www.eurosurveillance.org/ViewArticle.aspx?Articleld=20149

Child Health Information Systems (CHISs) are computerised clinical record systems which support a range of health promotion and prevention activities for children, including immunisation and screening. There are a number of different providers of CHISs in England. These systems are managed by child health departments in each local area and not all are interoperable. The establishment of systems which record and maintain accurate information on the entire population is critical to assess vaccination coverage at both national and local levels. These systems should have the flexibility to adapt to a continuously evolving immunisation programme, a mechanism to rapidly feedback to local public health teams for outbreak prevention and control, and the ability to mount a timely response to vaccine safety scares. The ability to schedule (call and recall) immunisation appointments has contributed to improvements in vaccination coverage both in England and elsewhere. While this has been achieved in England through multiple CHISs the development of a single national register would reduce the complexities of maintaining accurate and complete immunisation records for the entire population.

\section{Introduction}

The ability to reliably measure vaccine coverage plays an essential role in evaluating the success of a vaccination programme, identifying susceptible populations for further interventions and informing future vaccine policy decisions. This is dependent on having an accurate estimate for the eligible population (denominator) and a robust method of ascertaining the number of those eligible individuals who have received a particular vaccine dose (numerator).

Data on vaccines administered in England are currently recorded on two computerised systems - general practitioner (GP) registers and population-based child health information systems (CHISs). Similar systems operate across the United Kingdom (UK) (in Scotland, Wales and Northern Ireland) which enables the production of UK-wide estimates of vaccine coverage. In England, while CHISs are generally used to estimate vaccine coverage for the routine childhood immunisation programme, GP registers are often used to evaluate selective vaccination programmes for adults (e.g. seasonal influenza and pneumococcal polysaccharide vaccines). This paper will specifically focus on how data held on CHISs are used in England to assess the routine childhood immunisation programme in a timely and accurate manner.

The publication of the National Health Service (NHS) Health and Social Care Bill in 2011 marks a radical change to the organisational structure of the NHS in England [1]. The abolition of Primary Care Trusts (PCTs), currently responsible for maintaining CHISs, and changes to the responsibilities for the procurement of health services for local populations based on an assessment of their health needs are likely to have significant implications for the delivery and evaluation of national public health programmes including childhood immunisations. We also consider the challenges of collecting population-based vaccine coverage data through current systems in England, and of maintaining accurate collections in the newly structured NHS.

This paper focuses on how data held on CHISs have been used in England since the late 1980 s to assess vaccine coverage in the routine childhood immunisation programme in a timely and accurate manner, and considers the challenges of maintaining accurate collections in light of the planned reorganisation of the English National Health Service. It also highlights the lessons learnt from an English perspective which will be of relevance to those European countries planning to implement population-based immunisation registers.

\section{Child Health Information Systems (CHISs)}

CHISs are computerised clinical record systems which support a range of health promotion and prevention activities for children including screening and immunisation. There are a number of different providers of CHISs in England. These systems are managed by child health departments in each local area, previously in each district health authority and since the NHS 
reorganisation of April 2002, in Primary Care Trusts (PCTs).

They hold data on all children in the responsible population for that PCT. The PCT responsible population is defined as all children registered with a GP whose practice forms part of the $\mathrm{PCT}$, regardless of where the child is resident, plus any children not registered with a GP who are resident within the PCT's statutory geographical boundary. Note that children resident within the PCT geographical area, but registered with a GP belonging to another PCT, are the responsibility of that other PCT. Thus CHISs can provide a complete and accurate denominator for estimating vaccine coverage. Children are entered onto the local CHIS at birth or when they move into the local area. Much of the data required to start the child health record is already collected as part of the mother's maternal record stored in the local maternity information system. All new births are registered electronically by the attending midwife using the Central Issuing Service (CIS). This 'birth notification' automatically generates a unique NHS number within a few hours of delivery. An electronic copy of the birth notification containing core demographic information on the newborn and mother as well as GP registration details are sent from the CIS to the relevant Child Health department for entry onto the local CHIS. In addition, a paper record of the complete birth notification is faxed by the midwife to the local CHIS (Figure 1).
Records are transferred to the relevant CHIS for children moving into the area from other parts of the UK. When a child moves, he/she will register with a local GP who will request that all the child's health records (including their immunisation history) are transferred. Furthermore this practice will inform the local Child Health department managing the local CHIS of the newly-registered patient. A request is made to the former Child Health department to transfer their CHIS records. This transfer of information is either electronic or paper-based depending on the inter-operability of the different systems. For those children born outside the UK, a new record is created which includes all available data on vaccines that have been administered previously. Regular updating to take account of newborns and children moving in and out of the area is therefore essential. It is the responsibility of the PCT to ensure the accuracy of the data held on their local CHIS (Figure 1).

One of the primary functions of CHISs is to manage the local immunisation programme, scheduling appointments, recording data on vaccines administered and sending out reminders for those who fail to attend. Invitations for immunisation are either sent to parents / guardians from their registered GP or directly from the local CHIS. Information held on CHISs can be extracted to provide age-specific vaccine coverage estimates at local, regional and national levels. In addition, data held on CHISs are important for

\section{FIGURE 1}

Data flow to child health information system, England

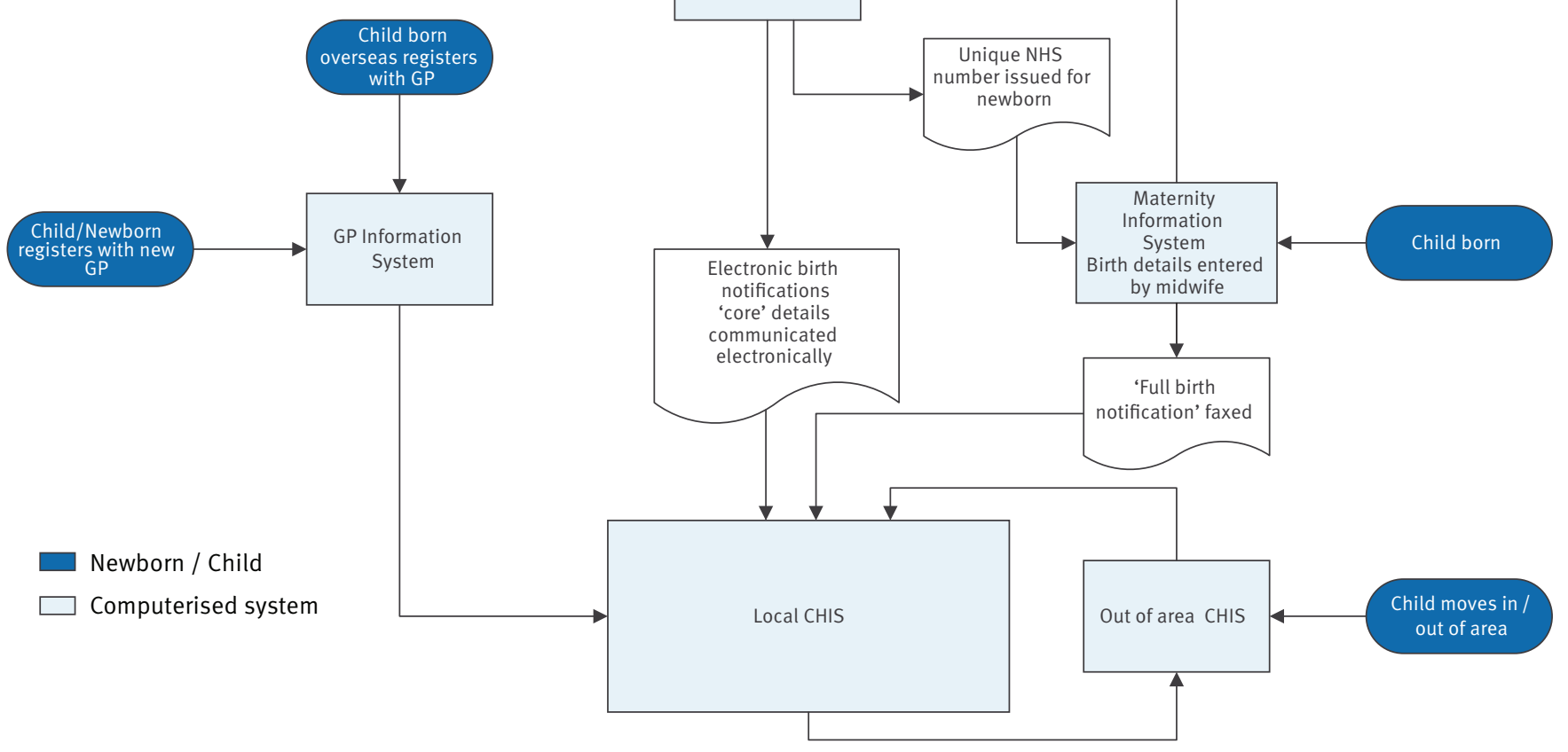


predicting and responding to community outbreaks of vaccine-preventable diseases (VPD) where local catchup programmes can be targeted at cohorts with poor coverage.

The majority of routine childhood immunisations are delivered to pre-school children by GPs in primary care although some selective programmes e.g. neonatal hepatitis B and Bacille Calmette Guerin (BCG) vaccines may be delivered in a combination of primary and secondary care settings. The contribution of vaccines delivered in the private sector currently is negligible. Vaccines administered in any of these settings are recorded electronically in the child's GP record and on the local CHIS. In addition, a paper copy of the children's vaccination record is held by the parent in the personal child health record (PCHR). Frequent exchange of information between CHISs, primary care and other providers is required to ensure assessment of vaccine coverage is based on accurate numerator and denominator estimates.

\section{Generating vaccine coverage data}

from CHISs: COVER Programme

Historically, long-term trends in childhood vaccine coverage in England were estimated by individual health authorities and published annually by the Department of Health (DH) [2]. Initially, the denominator was the number of live births in each district health authority, but in 1988, following the increasing use of computerised child health systems (CHISs), the denominator became all resident children in the district (Körner returns) [3]. Numerator data on the number of these eligible children receiving each of the recommended vaccines was also obtained from CHISs - providing, for the first time, a genuine measurement of total population coverage. Since 1995, annual vaccine coverage has been monitored by the Health Protection Agency (HPA) (previously Public Health Laboratory Service) on behalf of the $\mathrm{DH}$.

In addition to the annual collection, a quarterly collection was developed in the late-1980s to provide more rapid feedback and enable changes in vaccine coverage to be detected quickly [4]. This data collection system, known as the COVER programme (Cover of Vaccination Evaluated Rapidly) exploited the role of district immunisation co-ordinators as contacts and used standardised programmes to extract aggregate data from CHISs [4]. For a number of years, there has been a mandatory requirement for all local areas within the NHS to provide quarterly and annual returns to the HPA [5].

While the extraction processes may vary between the different CHISs, all system suppliers are provided with a specification detailing the 'request parameter's [6]. A quarterly request is made to each PCT Child Health department to provide computerised reports for these COVER parameters [7]. Information is requested for all children in the PCT responsible population who reach their first, second and fifth birthdays during a particular evaluation quarter. These data are aggregate returns and will include the number of eligible children in each cohort and the numbers and proportion vaccinated for all routine vaccinations offered according to the current national immunisation schedule (Figure 2).

The UK immunisation programme is constantly evolving. The addition of new vaccines and changes to the schedule requires CHISs to have the flexibility to incorporate these changes in a timely manner. At the time of planning the introduction of a new vaccine or change to the routine immunisation schedule, the DH works with all the CHIS suppliers to ensure that the systems can schedule and record data on new vaccines / schedule changes ahead of their implementation. These are communicated to the system suppliers through Dataset Change Notices (DSCNs). This in turn allows for the timely collection of vaccine coverage data on the first and subsequent cohorts eligible for the new schedule. Coverage data on the first eligible cohort following the introduction of the seven-valent pneumococcal conjugate vaccine to the primary infant schedule in September 2006 was published in December $2007[7,8]$.

\section{FIGURE 2}

Vaccine coverage data flows from Child Health Information Systems to the Health Protection Agency COVER programme, United Kingdom

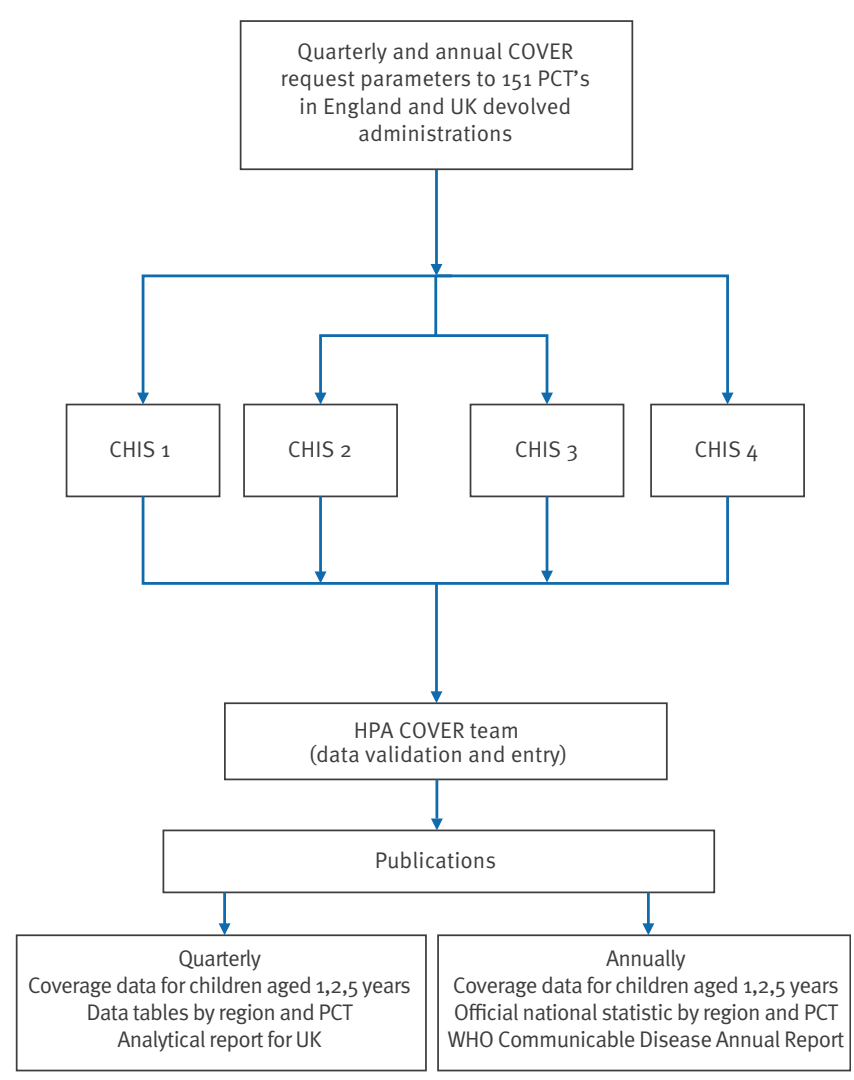

CHIS: Child Health Information System;

COVER: Cover of Vaccination Evaluated Rapidly,

HPA: Health Protection Agency;

PCT: Primary Care Trust;

UK: United Kingdom;

WHO: World Health Organization. 
Data quality requirements for vaccine coverage collections

The following section summarises the minimum data quality requirements for the collection of vaccine coverage data and the risks / implications if these requirements are not met.

\section{Accuracy and validation}

To ensure accuracy, precise definitions for the denominator (eligible population) and the numerator (number of individuals in the eligible population who have received a given vaccine(s) within a given time) are required. For the routine childhood immunisation programme, these parameters are published on the HPA website prior to each quarterly collection [6]. A number of checks are made as part of the data quality assurance process for each submission. These include verifying the evaluation period and comparing the denominator from the current evaluation quarter with the previous submissions to identify discrepancies. For example, unless there have been boundary changes, the denominator figures are unlikely to have changed significantly between evaluation periods. Significant variation in coverage estimates (+/- 5\%) from previous evaluation periods are also compared and investigated. Given that policy decisions and public health interventions at national and local level are informed by coverage data, inaccuracies in these data may result in inappropriate actions and the misuse of resources such as offering vaccines as part of a catch-up programme or a local outbreak response to individuals who are already fully protected.

Once the data has been collected, they are validated ('sense checking') prior to publication, within agreed timelines. This is essential to identify anomalies resulting from changes to the (i) national immunisation schedule (ii) Child Health Information Systems (CHISs) and (iii) vaccine preparations in use. There have sometimes been unexpected or temporary changes to vaccines offered due to vaccine shortages [9]. Awareness of these issues is imperative to understand the data and for the correct interpretation of current and future coverage estimates. In addition, variations in vaccine coverage for particular cohorts may result from national and local catch-up campaigns. This 'sense-checking' process requires historical knowledge and expertise of the UK immunisation schedule, an understanding of the complexities of CHISs and close working relationships with NHS staff providing these data.

\section{Completeness}

In contrast to sentinel systems, assessment of childhood vaccine coverage in England is a genuine measurement of total population coverage. In order to achieve this, data from each PCT are required and should be based on every eligible child. This is important to identify pockets of susceptible individuals who would benefit from targeted interventions.
Timeliness (collection and publication)

In England, vaccine coverage data are fed back promptly (within three months from the last evaluation quarter) to local public health teams, as provisional estimates, through the publication of UK COVER reports. These reports provide detailed commentary on the most recent coverage estimates at regional, national and UK level. Additionally, individual PCT level data for all vaccines assessed at one, two and five years of age are published on the HPA website, which allow national policy makers as well as local public health teams to consider appropriate interventions in a timely manner.

\section{Flexibility}

It may be necessary to undertake new /modified data collections in response to unexpected events. To assess the immediate impact of the adverse publicity surrounding the measles-mumps-rubella (MMR) vaccine in the UK, the COVER programme was able to set up a sentinel reporting system for monitoring MMR coverage from an earlier age and at more frequent intervals than routine collections [10]. This has provided a more timely indication of trends in MMR coverage, complementing the routine collections to inform vaccine policy decisions e.g. national MMR catch-up programme in 2008.

\section{Operational issues with CHISs}

\section{IT issues}

The number of system providers for CHISs and their functionality has expanded since their national rollout in the 1980s, necessitating replacement and / or upgrading of existing systems. Some CHISs have suffered from data quality issues as a result of these upgrades and the replacement of existing IT services $[11,12]$. Furthermore, the migration of data from legacy systems has made this a particular issue for older cohorts of children. In the past, the combination of different CHISs operating across London coupled with high population mobility made it difficult to maintain accurate data on each local system and has contributed to the lower coverage reported in the capital [13]. However, during the last five years, London PCTs have moved to the same system provider and have focused efforts on increasing coverage both through improving vaccine delivery and data quality [14]. While efforts have been made to ensure the exchange of information between systems is timely and complete, there is a need to ensure all current and future systems are fully interoperable.

\section{Denominator issues}

There is historic evidence to suggest that some CHISs were poorly maintained so that children who had moved away remained on the system. These 'ghosts' inflated the denominator and therefore led to an under-estimate of vaccine coverage. A review of eight unpublished audits of data held on CHISs in 1997 suggested that COVER data underestimated true uptake by between $1 \%$ and $9 \%$ in children assessed before 
their third birthdays [15]. Discrepancies increase when measuring coverage at five years given the increasing likelihood of moving PCTs with time, and fewer scheduled interventions which provide opportunities to identify children who have moved away/into the PCT. The greatest underestimates occurred in areas with lowest reported coverage and the highest population mobility.

Historically, NHS re-organisation has temporarily impacted on the quality of vaccine coverage data extracted from CHIS. For example, the last re-organisation in 2002, which coincided with a change in the definition of the denominator (from resident to responsible population), was thought to contribute to a reduction in the quality of COVER returns. This resulted in an underestimate of the denominator when compared with equivalent mid-year Office of National Statistics (ONS) population estimates [16] The lower denominator was partly explained by delays in including unregistered children who are less likely to access primary care services and have less opportunity to be vaccinated, leading to an overestimation of true coverage. Reassuringly, however, by 2003 , data quality improved as CHISs had begun to incorporate the reorganisational and population definition changes correctly [16].
Numerator issues

As the majority of routine childhood immunisations are delivered in GP, the accuracy of numerator data held on CHISs will be largely dependent on the accuracy and timeliness of the information supplied by GPs.

Although data held on CHISs are generally maintained until the age of 16 years, the accuracy of the numerator decreases with age given the reduced opportunities for older children and adolescents to routinely attend health services and for their records to be checked and updated. However, the introduction of the routine human papillomavirus (HPV) vaccination programme, targeting girls aged 12-13 years through a largely school-based programme, has provided a valuable opportunity to improve the accuracy of immunisation records for older children [17].

\section{Future considerations for monitoring vaccine coverage}

The radical reorganisation of NHS structures in England with the reallocation of local public health teams from the NHS to local government will necessitate maintaining the timely transfer of public health data, including vaccine coverage, across increasingly complex organisational boundaries.

\section{FIGURE 3}

Annual vaccination coverage at 24 months, England, 1987-2010

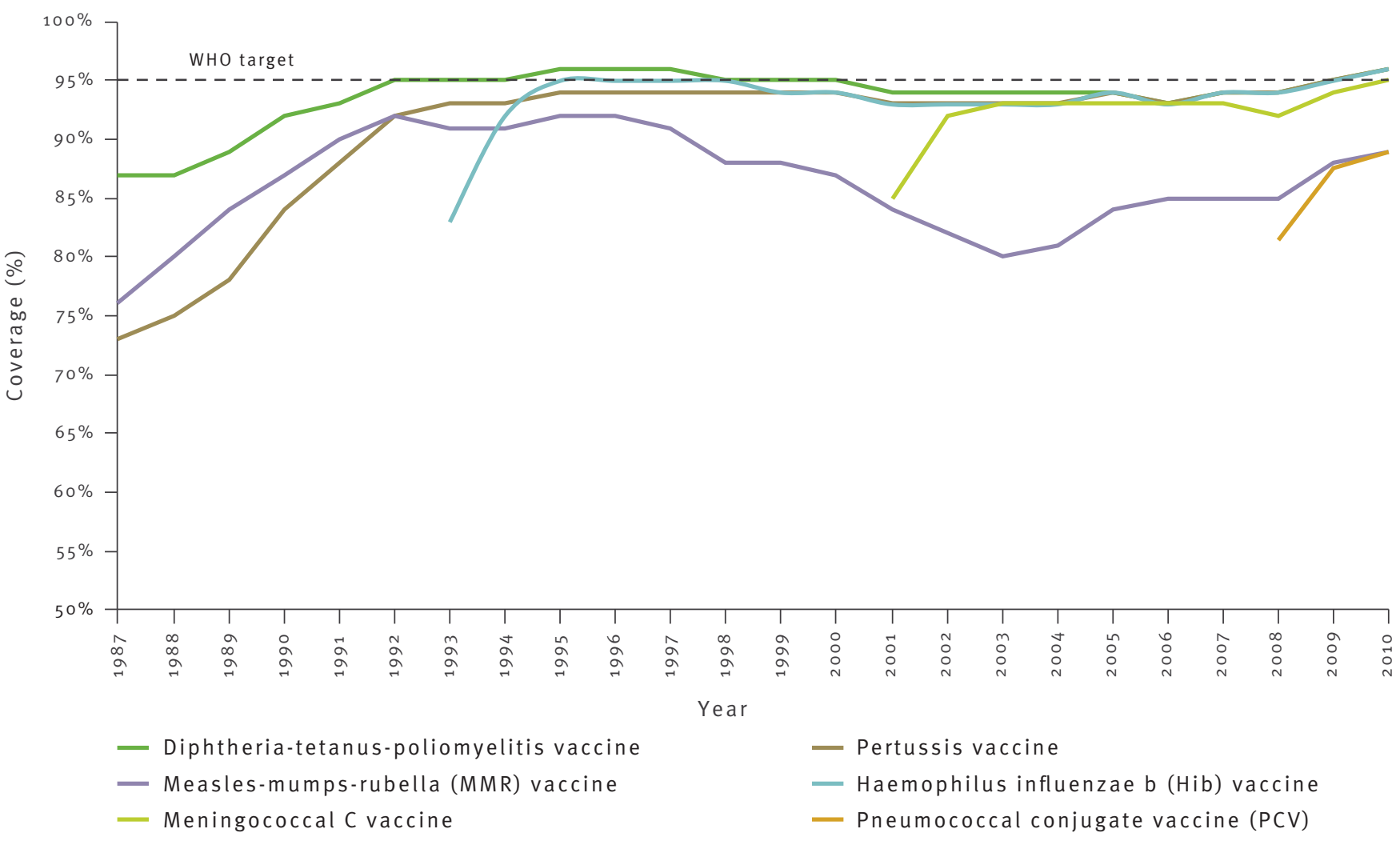


The abolition of PCTs and creation of Clinical Commissioning Groups (CCGs) with different geographical boundaries is likely to temporarily impact on the accuracy of denominator estimates and permanently impair the ability to compare trends in coverage data at a sub-national level. In addition, the potential increase in the number of non-NHS service providers will contribute to the challenges in maintaining accurate, up to date population based immunisation registers.

The challenge is to sustain the accurate and timely collection of vaccine coverage data to inform national policy decisions and local public health action. A programme of work led by the Department of Health, to address these issues, is currently underway with key stakeholders. This includes a proposal to agree a set of national minimum standards for CHISs that will deliver interoperable CHISs which can schedule appointments and communicate effectively with all provider systems.

\section{Implications for developing population- based immunisation registers in Europe} The UK has the longest running population-based child health registers in Europe that have contributed to a well organised and planned national immunisation programme. The system has survived many previous health service reorganisations in England, contributed to the achievement and maintenance of high vaccine coverage from the early 1990 s and helped to minimise the impact of adverse publicity in the early $2000 \mathrm{~s}$ (Figure 3).

Our experience has shown that there are a number of key requirements critical for success that may be relevant for European countries planning to introduce national immunisation registers. The establishment of a system which records and maintains accurate information on the entire population is critical to assess vaccination coverage at both national and local levels. These systems should have the flexibility to adapt to a continuously evolving immunisation programme, a mechanism to rapidly feedback coverage data to local public health teams for outbreak prevention and control, and the ability to mount a timely response to vaccine safety scares. The ability to schedule (call and recall) immunisation appointments has contributed to improvements in vaccination coverage both in England and elsewhere $[12,18]$. While this has been achieved in England through multiple CHISs which are not all interoperable, the development of a single national register would reduce the complexities of maintaining accurate and complete immunisation records for the entire population.
References

1. Department of Health (DH). Health and Social Care Bill 2011: combined impact assessments.. London: DH. [Accessed 13 Sep 2011]. Available from: http://www. dh.gov.uk/en/Publicationsandstatistics/Publications/ PublicationsLegislation/DH_123583

2. Health Protection Agency (HPA). Epidemiological data. London: HPA. [Accessed 12 Sep 2011]. Available from: http:// www.hpa.org.uk/Topics/InfectiousDiseases/InfectionsAZ/ VaccineCoverageAndCOVER/EpidemiologicalData/

3. Vyse AJ, Gay NJ, White JM, Ramsay ME, Brown DWG, Cohen BJ, et al. Evolution of Surveillance of Measles, Mumps and Rubella in England and Wales: Providing the Platform for Evidencebased Vaccination Policy. Epidemiol Rev. 2002;24(2):125-36.

4. Begg NT, Gill ON, White JM. COVER (Cover of Vaccination Evaluated Rapidly): Description of the England and Wales Scheme. Public Health. 1989;103(2):81-9.

5. NHS Information Centre for Health and Social Care. The Review of Central Returns (ROCR). London: NHS. Accessed 30 Sep 2011. Available from: http://www.ic.nhs.uk/rocr

6. Health Protection Agency (HPA). COVER Parameters. London: HPA. 12 May 2011. Available from: http://www. hpa.org.uk/Topics/InfectiousDiseases/InfectionsAZ/ VaccineCoverageAndCOVER/COVERParameters/

7. NHS Information Standards Board. Modification of existing vaccination and immunisation data return collected through the COVER (Cover of Vaccination Evaluated Rapidly) system in line with changes to the childhood immunisation programme. DSC Notice 21/2006. Sep 2006. Available from: http://www. isb.nhs.uk/documents/dscn/dscn2006/212006.pdf

8. Health Protection Agency (HPA). COVER programme: July to September 2007, including first evaluation of children at 12 months who were routinely offered pneumococcal vaccine (PCV) and data on the PCV catch-up programme. Health Protection Report. 2007;1(51). [Accessed 28 Feb 2012]. Available from: http://www.hpa.org.uk/hpr/archives/2007/ hpr5107.pdf

9. Department of Health. Letter from Chief Medical Officer, Chief Nursing Officer and Chief Pharmacist. Current vaccine issues: action update. 2 Dec 1999. Available from: http://www.dh.gov. uk/prod_consum_dh/groups/dh_digitalassets/@dh/@en/ documents/digitalasset/dh_4013402.pdf

10. Health Protection Agency (HPA). COVER Methods. London: HPA. [Accessed 28 Feb 2012]. Available from: http://www. hpa.org.uk/Topics/InfectiousDiseases/InfectionsAZ/ VaccineCoverageAndCOVER/COVERMethods/

11. NHS Information Centre for Health and Social Care. NHS Immunisation Statistics England. 2008-09. London: NHS. 3 Sep 2009. Available from: http://www.ic.nhs.uk/statisticsand-data-collections/health-and-lifestyles/immunisation/ nhs-immunisation-statistics-england-2008-09

12. Crowcroft NS. Action on immunisation: no data, no action. Arch Dis Child. 2009;94(11):829-30.

13. London Assembly. Health and Public Services Committee. London Assembly Reports. Still Missing the Point? Infant Immunisation in London. London Assembly. Sep 2007. Available from: http://legacy.london.gov.uk/assembly/reports/ health/infant_immunisation_followup.pdf

14. NHS Information Centre for Health and Social Care. NHS Immunisation Statistics, England 2009-10. November 2010. Available on-line at http://www.ic.nhs.uk/statistics-anddata-collections/health-and-lifestyles/immunisation/nhsimmunisation-statistics-england-2009-10 Accessed 30/09/11

15. Ramsay M, White J, Crowcroft N. MMR uptake data are unlikely to be subject to manipulation. BMJ. 2002;324(7350):1394.

16. Granerod J, White JM, Andrews N, Crowcroft NS. Vaccine Coverage in England: the impact of health service reorganisation. Arch Dis Child. 2006;91(10):805-7.

17. Health Protection Agency (HPA) / Department of Health (DH). Annual HPV vaccine coverage in England: 2009/2010. 22 Dec 2010. Available from http://www. dh.gov.uk/en/Publicationsandstatistics/Publications/ PublicationsPolicyAndGuidance/DH_123795

18. Jacobson Vann J, Szilagyi P. Patient reminder and patient recall systems to improve immunization rates. Cochrane Database Syst Rev. 2005;20(3): CD003941. 